\title{
AN ABSTRACT VERSION OF THE CONCENTRATION COMPACTNESS PRINCIPLE
}

\author{
I. SCHINDLER and K. TINTAREV
}

\begin{abstract}
We prove an abstract version of concentration compactness principle in Hilbert space and show its applications to a range of elliptic problems on unbounded domains.
\end{abstract}

\section{Introduction}

In the present paper we propose a generalization of the concentration compactness principle by P.-L.Lions $[9,10,11,12])$ from specific functional spaces to an abstact Hilbert space. One can define the concentration compactness aproach as a (partial) recovery of compactness in non-compact sequences by selecting certain, vaguely speaking, moving bulks of mass.

If $\alpha_{k} \in \mathbb{R}^{N}$ is an unbounded sequence and $u$ is a locally Lebesgueintegrable function on $\mathbb{R}^{N}$, the sequence $u_{k}:=u\left(\cdot-\alpha_{k}\right)$ will converge to zero in the sense of distributions. This means that nonzero shiftinvariant mappings are not weakly continuous. At the same time one can use weak convergence with different shifts to define a stronger topology in which some shift-invariant functionals become continuous. We say that a bounded sequence $u_{k}$ converges to zero weakly with concentration, if $u_{k}\left(\cdot-\alpha_{k}\right) \rightarrow 0$ for any sequence of shifts $\alpha_{k}$. As it is known, in the case of a Sobolev space over $\mathbb{R}^{N}$ concentrated weak convergence yields convergence in $L^{p}$ with subcritical $p$ (cf. [8]).

Instead of shifts we consider a general set of linear operators that might be called dislocations. This set cannot be arbitrarily small or arbitrarily large: if it is compact then concentrated weak convergence is 
the same as weak convergence, if it consists of all unitary operators, then the only sequences convergent weakly with concentration are constant sequences.

In Section 2 we prove, under certain assumptions about the set of dislocations, that any bounded sequence in a Hilbert space has a subsequence that can be written as an asymptotic sum of differently dislocated weak limits, with a remainder convergent to zero weakly with concentration. A tentative Banach space version has been proved in [18]. Applications of this decomposition require an analytic interpretation of concentrated weak convergence for specific choices of the space and the set of dislocations. Similar decompositions of sequences in Sobolev spaces have been in use since [13], but only under additional conditions on the sequence.

In Section 3 we deal with concretization of the abstract statement for Sobolev spaces with groups of shifts and dilations, and the product group thereof. The spaces invloved there are $H^{1}\left(\mathbb{R}^{N}\right)$ and $D^{1,2}\left(\mathbb{R}^{N}\right)$.

In Section 4 we study reduction of the above decompositions to Sobolev spaces over $\Omega \subset \mathbb{R}^{N}$. We introduce a class of open sets for which it is possible (asymptotically contractive sets) and give their geometric characterization. At the end of the section we give two examples of compactness results for Sobolev imbeddings.

Section 5 presents applications of the method to semilinear elliptic equations. We provide existence results for: a model problem on an asymptotically contractive set; a problem on exterior domain with symmetry; a problem with a critical exponent on unbounded asymptotically contractive domains.

\section{Abstract concentration compactness in Hilbert space}

Let $H$ be a separable Hilbert space and let $D$ be a bounded set of bounded linear operators on $H$, closed with respect to strong convergence and satisfying the following properties:

(I) $g \in D \Rightarrow g^{-1} \in D, I \in D$;

(II) if $g_{k}, h_{k} \in D$ and $g_{k} h_{k}^{-1}$ does not converge weakly to 0 , then there exists a renamed strongly convergent subsequence of 


$$
g_{k} h_{k}^{-1} \text { such that } \mathrm{s}-\lim g_{k} h_{k}^{-1} \neq 0
$$

(III) $g_{k} \in D, u_{k} \rightarrow 0 \Rightarrow g_{k}^{*} g_{k} u_{k} \rightarrow 0$.

We shall call $D$ a set of dislocations. Dislocations do not have to form a group, however, for a group of unitary operators one can replace conditions (I-III) by a single condition as below.

Proposition 2.1. Let $D$ be a group of unitary operators in $H$. Then $D$ is a set of dislocations if the following condition is satisfied:

(IIa) if $g_{k} \in D$ does not converge weakly to zero, then there exists a renamed strongly convergent subsequence of $g_{k}$ such that $\mathrm{s}-\lim g_{k} \neq$ 0 .

Proof. Condition (I) follows from the definition of a group. Condition (III) is tautological since $D$ consists of isometries and $g_{k}^{*} g_{k}=i d$. Condition (II) rewritten for a group becomes (IIa).

Definition 2.2. Let $u, u_{k} \in H$. We will say that $u_{k}$ converges to $u$ weakly with concentration (with respect to a given set of dislocations $D)$, which we will denote as

$$
u_{k} \stackrel{c w}{\rightarrow} u
$$

if for all $\varphi \in H$,

$$
\lim _{k \rightarrow \infty} \sup _{g \in D}\left(g\left(u_{k}-u\right), \varphi\right)=0
$$

The goal of this section is to prove the theorem on decomposition of sequences into dislocated weak limits:

Theorem 2.3. Let $u_{k} \in H$ be a bounded sequence and let $D$ be a set of dislocations. Then there exist $w^{(n)} \in H, g_{k}^{(n)} \in D, k, n \in \mathbb{N}$ such that for a renumbered subsequence

$$
\begin{gathered}
w^{(n)}=\mathrm{w}-\lim g_{k}^{(n)^{-1}} u_{k}, \quad g_{k}^{(1)}=i d, \\
g_{k}^{(n)^{-1}} g_{k}^{(m)} \rightarrow 0 \text { for } n \neq m,
\end{gathered}
$$




$$
\sum_{n \in \mathbb{N}}\left\|w^{(n)}\right\|^{2} \leq \limsup \left\|u_{k}\right\|^{2}
$$

and

$$
u_{k}-\sum_{n \in \mathbb{N}} g_{k}^{(n)} w^{(n)} \stackrel{c w}{\rightarrow} 0
$$

wihere the series is absolutely convergent for every $k$.

Proof. We define, on a renumbered subsequence,

$$
w^{(1)}=: \mathrm{w}-\lim u_{k}, g_{k}^{(1)}=i d .
$$

Let

$$
v_{k}^{(1)}=u_{k}-g_{k}^{(1)} w^{(1)}
$$

If $v_{k}^{(1)} \stackrel{c w}{\rightarrow} 0$, the theorem is verified with $w^{(n)}=0, n \geq 2$. If not, there exist $g_{k}^{(2)}$ and $w^{(2)} \neq 0$ such that, on a renumbered subsequence,

$$
g_{k}^{(2)^{-1}} v_{k}^{(1)} \rightarrow w^{(2)} .
$$

If $g_{k}^{(1)^{-1}} g_{k}^{(2)} \nrightarrow 0$, then, applying this product to (8), and using (II), we obtain for a renamed subsequence,

$$
g_{k}^{(1)^{-1}} v_{k}^{(1)}-g_{k}^{(1)^{-1}} g_{k}^{(2)} w^{(2)} \rightarrow 0 .
$$

Thus, on a renamed subsequence, noting that due to (I) $\lim g_{k}^{(1)^{-1}} g_{k}^{(2)} w^{(2)} \neq 0$, we have:

$$
\mathrm{w}-\lim g_{k}^{(1)^{-1}} v_{k}^{(1)} \neq 0 .
$$

However, using (7) and (6), we get

$$
\mathrm{w}-\lim g_{k}^{(1)^{-1}} v_{k}^{(1)}=\mathrm{w}-\lim g_{k}^{(1)}{ }^{-1} u_{k}-w^{(1)}=0 .
$$

This contradiction implies that

$$
g_{k}^{(1)^{-1}} g_{k}^{(2)} \rightarrow 0,
$$

Recursively we define:

$$
v_{k}^{(n)}=u_{k}-g_{k}^{(1)} w^{(1)}-\cdots-g_{k}^{(n)} w^{(n)}
$$


If for some $n, v_{k}^{(n)} \stackrel{c w}{\longrightarrow} 0$, the statement is proved. Otherwise, there is $g_{k}^{(n+1)}$, and a $w^{(n+1)} \neq 0$ such that, on a subsequence

$$
g_{k}^{(n+1)^{-1}} v_{k}^{(n)} \rightarrow w^{(n+1)} .
$$

An argument similar to that above shows that

$$
g_{k}^{(p)-1} g_{k}^{(q)} \rightarrow 0 \text { whenever } p \neq q .
$$

From (13),(14),(15) follows (2). Let us now expand the left hand side of the inequality $\left\|v_{k}^{(n)}\right\|^{2} \geq 0$ using (13):

$$
\begin{array}{r}
\left\|u_{k}\right\|^{2}+\sum_{n}\left\|g_{k}^{(n)} w^{(n)}\right\|^{2}-2 \sum_{n}\left(u_{k}, g_{k}^{(n)} w^{(n)}\right)+\sum_{m \neq n}\left(g_{k}^{(m)} w^{(m)}, g_{k}^{(n)} w^{(n)}\right) \\
\geq 0
\end{array}
$$

The last term in (16) converges to zero due to (15) and (III), since $g_{k}^{(n)^{*}} g_{k}^{(m)}=\left[g_{k}^{(n)^{*}} g_{k}^{(n)}\right]\left[g_{k}^{(n)^{-1}} g_{k}^{(m)}\right]$. Let us estimate the third term.

$$
\begin{array}{rrr}
\sum_{n}\left(u_{k}, g_{k}^{(n)} w^{(n)}\right)= & \sum_{n}\left(g_{k}^{(n)^{*}} g_{k}^{(n)} g_{k}^{(n)^{-1}} u_{k}, w^{(n)}\right) \\
= & \sum_{n}\left(g_{k}^{(n)^{*}} g_{k}^{(n)}\left(g_{k}^{(n)^{-1}} u_{k}-w^{(n)}\right), w^{(n)}\right) \\
+\sum_{n}\left(g_{k}^{(n)^{*}} g_{k}^{(n)} w^{(n)}, w^{(n)}\right) \\
= & o(1)+\sum_{n}\left(g_{k}^{(n)^{*}} g_{k}^{(n)} w^{(n)}, w^{(n)}\right) .
\end{array}
$$

in the last step we have applied (III) and (14). Combining (16) and (17) we get:

$$
\left\|u_{k}\right\|^{2} \geq \sum_{n}\left\|g_{k}^{(n)} w^{(n)}\right\|^{2}+o(1)
$$

Due to (I) we can deduce from (18) that

$$
\sum_{n}\left\|w^{(n)}\right\|^{2} \leq C \lim \sup \left\|u_{k}\right\|^{2}, C>0
$$


It is easy to see, using (19) and (15), that the series $\sum g_{k}^{(n)} w^{(n)}$ (after passing to an appropriately rarefied subsequence) is absolutely convergent. Let $\epsilon>0$. Note that by (19) there is a $M>0$, independent of the selection of $w^{(n)}$ 's such that the number of weak limits (14) with the norm exceeding $\epsilon / 2$ is less than $M$. This implies that if on every iteration step we will be selecting a weak limit with the norm exceeding $\epsilon / 2$ (if there is any), then for every $g_{k} \in D$, any weak subsequential limit of $g_{k}^{-1} v_{k}^{(M)}$ will have a norm less than $\epsilon$.

Let $\varphi \in H,\|\varphi\|=1$ and $g_{k} \in D$. Then

$$
\limsup \left|\left(g_{k}^{-1} v_{k}^{(M)}, \varphi\right)\right| \leq 2\left|\left(w^{(M+1)}, \varphi\right)\right| \leq 2\left\|w^{(M+1)}\right\| \leq 2 \epsilon
$$

And so

$$
\limsup \left|\left(g_{k}^{-1}\left(u_{k}-\sum_{n} g_{k}^{(n)} w^{(n)}\right), \varphi\right)\right|=0,
$$

which verifies (5).

\section{Concentration compactness in Sobolev spaces}

Assume first that $H=H_{0}^{1}\left(\mathbb{R}^{N}\right)$ and that $D$ is a group of shift operators $g_{\alpha} u=u(\cdot+\alpha), \alpha \in \mathbb{R}^{N}$. Shifts are unitary operators on $H$ and it is easy to see that they verify condition (IIa). First,

$$
g_{\alpha_{k}} \rightarrow 0 \Leftrightarrow\left|\alpha_{k}\right| \rightarrow \infty .
$$

A bounded sequence $\alpha_{k}$ has a convergent subsequence, so that $u_{k} \rightarrow$ $0 \Rightarrow\left(g_{\alpha_{k}} u_{k}, \varphi\right)=\left(u_{k}, g_{-\alpha_{k}} \varphi\right)$, and the latter expression has the same $\operatorname{limit}$ as $\lim \left(u_{k}, g_{-\lim \alpha_{k}} \varphi\right)=0$. Theorem 2.3 now can be quoted together with (22) while the analytical meaning of concentrated weak convergence is clarified by the following statement. We will use the following notation: $2^{*}=\frac{2 N}{N-2}$ for $N>2$ and $2^{*}=\infty$ when $N=2$.

Lemma 3.1. Let $u_{k}$ be a bounded sequence in $H^{1}\left(\mathbb{R}^{N}\right)$ and $p \in\left(2,2^{*}\right)$. Then $u_{k} \stackrel{c w}{\rightarrow} 0 \Leftrightarrow\left\|u_{k}\right\|_{L^{p}\left(\mathbb{R}^{N}\right)} \rightarrow 0$.

Proof. The if implication is an elementary corollary of Lemma 6 in [8] and can be also found in [10]. For the only if statement note that due 
to the shift invariance of $L^{p}$-norm, all $L^{p}$-weak limits of sequences $g_{k} u_{k}$ are equal to zero. Since the sequence $u_{k}$ is bounded $H^{1}$-norm, $L^{p}$-weak convergence implies weak convergence in $H^{1}$ and thus, concentrated weak convergence.

An immediate consequence of this lemma is

Lemma 3.2. Let $H=H^{1}\left(\mathbb{R}^{N}\right)$, let $D$ be the group of shifts and let $u_{k}, w^{(n)}$, and $g_{k}^{(n)}$ be as in Theorem 2.3. If $F: \mathbb{R} \rightarrow \mathbb{R}$ is a continuous function and for every $\epsilon>0$ there is a $C_{\epsilon}<\infty$ and a $p_{\epsilon}$ such that $2<p_{\epsilon}<2^{*}$ and

$$
|F(s)| \leq \epsilon\left(|s|^{2}+|s|^{2^{*}}\right)+C_{\epsilon}|s|^{p_{\epsilon}}
$$

then

$$
\lim _{k \rightarrow \infty} \int_{\mathbb{R}^{N}} F\left(u_{k}\right)=\sum_{n} \int_{\mathbb{R}^{N}} F\left(w^{(n)}\right) .
$$

Proof. It is easy to see from Lemma 3.1 and (23) that $u \rightarrow \int_{\mathbb{R}^{N}} F(u)$ is a continuous map with respect to concentrated weak convergence. This reduces the proof to the case of finite sums, i.e. one can assume without loss of generality that $u_{k}=\sum_{n=1}^{M} w^{(n)}\left(\cdot+\alpha_{k}^{(n)}\right)$. Note that by (22) and (3), $\left|\alpha_{k}^{(n)}-\alpha_{k}^{(m)}\right| \rightarrow \infty$ for $m \neq n$, so that for every $\epsilon>0$ one can find $R>0$ and $k_{0} \in \mathbb{N}$ such that for all $k>k_{0}$ the balls $B_{R}\left(-\alpha_{k}^{(n)}\right)$ will be disjoint and for $p=2,2^{*}$ one will have

$$
\int_{\mathbb{R}^{N} \backslash B_{R}\left(-\alpha_{k}^{(n)}\right)}\left|w^{(n)}\right|^{p} \leq \epsilon,
$$

as well as

$$
\int_{\mathbb{R}^{N} \backslash B_{R}\left(-\alpha_{k}^{(n)}\right)}\left|w^{(m)}\right|^{p} \rightarrow 0 \text { for } m \neq n \text { as } k \rightarrow \infty .
$$

This together with (23) allows, with an error of order $\epsilon$, to reduce integration to the balls $B_{R}\left(-\alpha_{k}^{(n)}\right)$, where the contribution of the terms $w^{(m)}\left(\cdot+\alpha_{k}^{(m)}\right), m \neq n$, will go to zero.

In the space $H^{1}\left(\mathbb{R}^{N} ; \mathbb{C}\right)$ one can also consider a set of dislocations of the following form:

$$
g_{\alpha} u(x)=e^{i \Phi(\alpha, x)} u(x+\alpha)
$$


with a $C^{1}$-function $\Phi$ that is odd in $\alpha$ and has a uniformly bounded gradient. This set is not a group, but it satisfies (I-III), and the concentrated weak convergence in this case implies $L^{p}$-convergence as well. This requires only trivial modifications in the proof of Lemma 3.1. Lemma 3.2 will be also true if one assumes that $F$ is dependent on $|u|$ alone.

We now let $H=D^{1,2}\left(\mathbb{R}^{N}\right), N>2$, and let $D$ be the product of the group of shifts $g_{\alpha}$ and the group of dilations:

$$
h_{t} u(x):=t^{\frac{N-2}{2}} u(t x), t \in(0, \infty) .
$$

Note that every element of $D$ can be represented as $u \mapsto h_{t} u(\cdot+$ $\alpha$ ) with some $\alpha \in \mathbb{R}^{N}$ and $t>0$ and that it is a unitary operator. Verification of the property (IIa) is similar to the case of $H^{1}$ with shifts, if one takes into account that

$$
h_{t_{k}}\left(\cdot+\alpha_{k}\right) \rightarrow 0 \Leftrightarrow t_{k} \rightarrow 0 \text { or } t_{k} \rightarrow \infty \text { or }\left|\alpha_{k}\right| \rightarrow \infty .
$$

Therefore Theorem 2.3 applies also in this case.

In many applications one can benefit from using Theorem 2.3 in a larger space and then study the restriction to the original space. We apply this approach below to restrictions from $D^{1,2}$ to $H^{1}$, while in the next section we will study restrictions to $H_{0}^{1}(\Omega), \Omega \subset \mathbb{R}^{N}$.

Lemma 3.3. Let $H$ and $D$ be as above and assume the conditions of Theorem 2.3 and moreover, that

$$
\left\|u_{k}\right\|_{L^{2}\left(\mathbb{R}^{N}\right)} \leq C
$$

with $C>0$. Then to the assertions of Theorem 2.3 one can add that in the terms $g_{k}^{(n)} w^{(n)}:=h_{t_{k}^{(n)}} w^{(n)}\left(\cdot+\alpha_{k}^{(n)}\right)$ none of the dilation parameters $t_{k}^{(n)}$ will converge to zero and that for every $n$ such that $t_{k}^{(n)}$ is bounded, $w^{(n)} \in H^{1}\left(\mathbb{R}^{N}\right)$.

Proof. When $t_{k}^{(n)}$ is bounded, due to Theorem 2.3,

$$
w^{(n)}:=\mathrm{w}-\lim \left(h_{1 / t_{k}^{(n)}} u_{k}\right)\left(\cdot-\alpha_{k}^{(n)}\right),
$$

and since the right hand side is bounded in $L^{2}$, the function $w^{(n)}$ is a weak limit also in the sense of $H^{1}$. Without loss of generality assume 
that $w^{(n)} \neq 0$. If $t_{k}^{(n)} \rightarrow 0$, the $L^{2}$ norm of the sequence in the right hand side of (31) will converge to zero, which implies (by Fatou lemma) that $\left\|w^{(n)}\right\|_{L^{2}\left(\mathbb{R}^{N}\right)}=0$, a contradiction.

When the set of dislocations includes dilations, concentrated weak convergence implies also convergence in $L^{2^{*}}$.

Lemma 3.4. Let $D$ be as above and let $u_{k}$ be a bounded sequence in $H^{1}\left(\mathbb{R}^{N}\right), N>2$. If $u_{k} \stackrel{c w}{\rightarrow} 0$, then $\left\|u_{k}\right\|_{L^{p}} \rightarrow 0$ for $2<p \leq 2^{*}$.

Proof. For $2<p<2^{*}$ this follows from Lemma 3.1. Consider now the case $p=2^{*}$. Let $t_{k} \rightarrow \infty$ be such that

$$
\int_{\left|u_{k}\right|>t_{k}^{\frac{N-2}{2}}}\left|u_{k}\right|^{2^{*}} \rightarrow 0
$$

Let $v_{k}$ be defined by

$$
u_{k}=t_{k}^{\frac{N-2}{2}} v_{k}\left(t_{k} x\right)
$$

Then

$$
\int_{\left|u_{k}\right|<t_{k}^{\frac{N-2}{2}}}\left|u_{k}\right|^{2^{*}}=\int_{v_{k}<1}\left|v_{k}\right|^{2^{*}} \leq \int_{\mathbb{R}^{N}}\left|v_{k}\right|^{2+\frac{1}{N}} \rightarrow 0,
$$

since $v_{k} \stackrel{c w}{\longrightarrow} 0$ and $2<2+\frac{1}{N}<2^{*}$. Relation (32) combined with (34) verifies the lemma.

Remark 3.5. We would like to remark that all terms in (5) with their dilation parameters $t_{k}^{(n)}$ and their reciprocal values $t_{k}^{(n)^{-1}}$ bounded, can be replaced, on a renumbered subsequence, with terms of the form $w^{(n)}\left(\cdot+\alpha_{k}^{n}\right)$.

Lemma 3.6. Let $u_{k}$ satisfy the assumptions of Lemma 3.3 and let $w^{(n)}$, $\alpha_{k}^{(n)}$, and $t_{k}^{(n)}$ be as provided by the lemma. If $F: \mathbb{R} \rightarrow \mathbb{R}$ is a continuous function satisfying (23) then

$$
\lim _{k \rightarrow \infty} \int_{\mathbb{R}^{N}} F\left(u_{k}\right)+\left|u_{k}\right|^{2^{*}}=\sum_{n \in \mathbb{N}_{1}} \int F\left(w^{(n)}\right)+\sum \int\left|w^{(n)}\right|^{2^{*}}
$$

where $\mathbb{N}_{1}$ consists of all indices $n$ such that $t_{k}^{(n)}$ is bounded. 
Proof. When $n \notin \mathbb{N}_{1}, t_{k}^{\frac{N-2}{2}} w^{(n)}\left(t_{k}^{(n)}\left(\cdot+\alpha_{k}^{(n)}\right)\right) \rightarrow 0$ in $L^{p}, 2<p<2^{*}$. Thus due to Lemma 3.4 and using Remark 3.5,

$$
u_{k}-\sum_{n \in \mathbb{N}_{1}} w^{(n)}\left(\cdot+\alpha_{k}^{(n)}\right) \rightarrow 0 \text { in } L^{p}, 2 \leq p<2^{*}
$$

with $\left|\alpha_{k}^{(n)}-\alpha_{k}^{(m)}\right| \rightarrow \infty$ for $n \neq m ; n, m \in \mathbb{N}_{1}$. Thus, by Lemma 3.2 (once one notices that the proof of the lemma uses the asymptoptics of (2.3) as $L^{p}$-asymptotics),

$$
\lim _{k \rightarrow \infty} \int_{\mathbb{R}^{N}} F\left(u_{k}\right)=\sum_{n \in \mathbb{N}_{1}} \int_{\mathbb{R}^{N}} F\left(w^{(n)}\right) .
$$

The proof that

$$
\int\left|u_{k}\right|^{2^{*}} \rightarrow \sum_{n \in \mathbb{N}} \int\left|w^{(n)}\right|^{2^{*}}
$$

is based on the conclusion of Lemma 3.4:

$$
u_{k}-\sum_{n} t_{k}^{(n) \frac{N-2}{2}} w^{(n)}\left(t_{k}^{(n)}\left(\cdot+\alpha_{k}^{(n)}\right)\right) \rightarrow 0 \text { in } L^{2^{*}}
$$

with either $t_{k}^{(n)} / t_{k}^{(m)} \rightarrow \infty$ or $t_{k}^{(m)} / t_{k}^{(n)} \rightarrow \infty$ or $\left|\alpha_{k}^{(n)}-\alpha_{k}^{(m)}\right| \rightarrow \infty$ for all $m \neq n$, which leads to separation of the contributions of different terms to the integral. The details are left to the reader.

\section{Traces of dislocated weak limits on $H_{0}^{1}(\Omega)$}

Let $\Omega \subset \mathbb{R}^{N}$ be an open set. In this section we consider the question when, for a bounded sequence $u_{k} \in H_{0}^{1}(\Omega)$, extended to $\left.H^{(} \mathbb{R}^{N}\right)$ one gets dislocated weak limits $w^{(n)}$ in Theorem 2.3, that can be identified as elements of $H_{0}^{1}(\Omega)$. We start with directly defining a class of open sets by this property (asymptotically contractive sets) and then give two sufficient geometric conditions of asymptotic contractiveness.

At the end of the section we give two examples of compactness in Sobolev embeddings. 


\subsection{Asymptotically contractive sets}

Definition 4.1. An open set $\Omega \subset \mathbb{R}^{N}$ will be called asymptotically contractive if for every sequence $u_{k} \in H_{0}^{1}(\Omega)$ and every sequence $\alpha_{k} \in$ $\mathbb{R}^{N}$ such that $u_{k}\left(\cdot+\alpha_{k}\right)$ converges weakly in $H^{1}\left(\mathbb{R}^{N}\right)$ to some $w$, there exists a $\gamma \in \mathbb{R}^{N}$ such that $w(\cdot-\gamma) \in H_{0}^{1}(\Omega)$.

An open set $\Omega \subset \mathbb{R}^{N}$ will be called asymptotically null if for every sequence $u_{k} \in H_{0}^{1}(\Omega)$ and every sequence $\alpha_{k} \in \mathbb{R}^{N},\left|\alpha_{k}\right| \rightarrow \infty, u_{k}(\cdot+$ $\left.\alpha_{k}\right) \rightarrow 0$.

This definition allows to formulate following statements:

Proposition 4.2. Let $H=H^{1}\left(\mathbb{R}^{N}\right)$, let $D$ be the group of shifts. If $\Omega \subset \mathbb{R}^{N}$ is an asymptotically contractive open set and $u_{k} \in H_{0}^{1}(\Omega)$, then the conclusions of Theorem 2.3 hold with $w^{(n)} \in H_{0}^{1}(\Omega), n \in \mathbb{N}$.

Proposition 4.3. Let $H=H^{1}\left(\mathbb{R}^{N}\right)$, let $D$ be the product group of shifts and dilations as defined in Section 3. If $\Omega \subset \mathbb{R}^{N}$ is an asymptotically contractive open set and $u_{k} \in H_{0}^{1}(\Omega)$, then the conclusions of Theorem 2.3 hold with $w^{(n)} \in H_{0}^{1}(\Omega)$ whenever $n \in \mathbb{N}_{1}$.

\subsection{Geometric characterization of asymptotically contrac- tive sets}

Let $\alpha_{k} \in \mathbb{R}^{N}, u_{k} \in D^{1,2}\left(\mathbb{R}^{N}\right)$ and let $u_{k}\left(\cdot-\alpha_{k}\right) \rightarrow w$. Then there exists a set $Y \subset \mathbb{R}^{N}$ of measure zero such that for all $x \notin Y, u_{k}\left(x-\alpha_{k}\right) \rightarrow w(x)$. Then, if for a given $x \notin Y$ there is a subsequence where $u_{k}\left(x-\alpha_{k}\right)=0$, then $w(x)=0$. In particular, if $u_{k} \in H_{0}^{1}(\Omega)$, where $\Omega \subset \mathbb{R}^{N}$ is an open set, then whenever $x \notin \Omega+\alpha_{k}$ holds on a subsequence, $w(x)=0$. We have to note only that one has to replace $u_{k}$ by their $C_{0}^{\infty}$-approximations in the gradient norm, so that questions about trace on the boundary will not arise. We conclude that except for points in $Y, w$ is non-zero only for such $x$ that $x \in \Omega+\alpha_{k}$ for all $k$ sufficiently large, that is, for

$$
x \in \liminf \left(\Omega+\alpha_{k}\right):=\bigcup_{n \in \mathbb{N}} \bigcap_{k \geq n}\left(\Omega+\alpha_{k}\right) .
$$

Lemma 4.4. Let $u_{k} \in H_{0}^{1}(\Omega), \alpha_{k} \in \mathbb{R}^{N}$ and let $u_{k}\left(\cdot+\alpha_{k}\right) \rightarrow w$. Then there exist a set $C$ of zero capacity and a set $Y$ of zero measure such that

$$
x \notin \liminf \left(\Omega+\alpha_{k}\right) \cup Y \Rightarrow w(x)=0,
$$




$$
x \notin \lim \sup \left(\Omega+\alpha_{k}\right) \cup C \Rightarrow w(x)=0
$$

Proof. The first assertion of the lemma follows from (40). The proof of the second assertion is similar if one notices that there are convex combinations of $u_{k}\left(\cdot-\alpha_{k}\right)$ that converge in norm and thus quasi-everywhere, so that $w(x) \neq 0$ if $x$, apart from a set of zero capacity, is not in the union of translated $\Omega$ over any tail of the sequence, i.e. not in $\left.\lim \sup \left(\Omega+\alpha_{k}\right)\right):=\bigcap_{n \in \mathbb{N}} \bigcup_{k \geq n}\left(\Omega+\alpha_{k}\right)$. Subadditivity of capacity implies (42).

Lemma 4.5. Let $\Omega \subset \mathbb{R}^{N}$ be an open set, let $u_{k} \in H_{0}^{1}\left(\mathbb{R}^{N}\right)$,

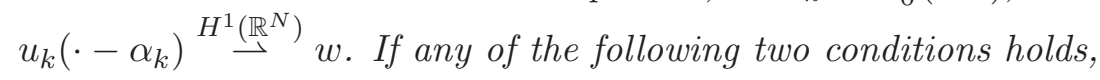

(i) there is a set $Y$ of measure 0 and a sequence $\gamma_{k} \in \mathbb{R}^{N}, \gamma_{k} \rightarrow 0$, such that $\liminf \left(\Omega+\alpha_{k}-\gamma_{k}\right) \subset \Omega \cup Y$ and $\partial \Omega=\partial\left(\mathbb{R}^{N} \backslash \bar{\Omega}\right)$, or

(ii) there exists a subsequence $\left\{\beta_{k}\right\} \subset\left\{\alpha_{k}\right\}$, a sequence $\gamma_{k} \in \mathbb{R}^{N}, \gamma_{k} \rightarrow$ 0 , and a set $C$ of capacity 0 such that $\lim \sup \left(\Omega+\beta_{k}-\gamma_{k}\right) \subset \Omega \cup C$, then $w \in H_{0}^{1}(\Omega)$.

Proof. Without loss of generality we can assume that $\gamma_{k}=0$ since $z_{k}\left(\cdot-\gamma_{k}\right)-z_{k} \rightarrow 0$ for any bounded sequence $\gamma_{k}$.

Indeed, let $v \in C_{0}^{\infty}\left(\mathbb{R}^{N}\right)$. Then

$$
\left|\left(z_{k}\left(\cdot-\gamma_{k}\right)-z_{k}, v\right)\right| \leq\left\|z_{k}||\right\| \mid v\left(\cdot+\gamma_{k}\right)-v \| \rightarrow 0 .
$$

We consider $w$ refined and thus defined quasi-everywhere (i.e., modulo sets of capacity zero. For definitions and statements from potential theory used in this proof we refer the reader to [1], cf. also [14].) Under condition (i), $w=0$ a.e. on the open set $\mathbb{R}^{N} \backslash \bar{\Omega}$ implies that $w=0$ q.e. on the closure of that set. Under the geometric assumption of (i) $w=0$ q.e. in the complement of $\Omega$. Same conclusion results from (ii) directly. The assertion of the theorem follows immediately from the Hedberg trace theorem.

Remark 4.6. We would like to formulate a conjecture that both conditions (i) and (ii) of Lemma 4.5 can be replaced by a milder sufficient condition

$$
\exists \gamma_{k} \rightarrow 0, \liminf \left(\Omega+\alpha_{k}-\gamma_{k}\right) \subset \Omega \cup Y .
$$


This would be true if the weak convergence in $H^{1}$ implied convergence quasi-everywhere. However, the latter statetement to our best knowledge is itself an open conjecture.

Lemma 4.5 provides geometric sufficient conditions for an open set to be asymptotically contractive:

Corollary 4.7. An open set $\Omega \subset \mathbb{R}^{N}$ is asymptotically contractive, if for every sequence $\alpha_{k} \in \mathbb{R}^{N}$ there is a renumbered subsequence and a $\gamma \in \mathbb{R}^{N}$ such that any of two conditions (i), (ii) of Lemma 4.5, with $\gamma_{k} \rightarrow 0$ replaced by $\gamma_{k} \rightarrow \gamma$, holds.

Corollary 4.8. The following sets are asymptotically contractive:

(i) An asymptotically null set (in particular any open set $\Omega$ such that whenever $\left|\alpha_{k}\right| \rightarrow \infty,\left|\liminf \left(\Omega+\alpha_{k}\right)\right|=0$, e.g. a bounded open set);

(ii) An open set $\Omega \subset \mathbb{R}^{N}$ such that for every $\alpha \in \mathbb{Z}^{N}, \Omega+\alpha \subset \Omega$ (and in particular, $\mathbb{R}^{N}$ );

(iii) An open set $\Omega \subset \mathbb{R}^{N}$ such that for certain $M \in \mathbb{N}, M<N$, and for every $\alpha \in \mathbb{Z}^{M}, \Omega+\alpha=\Omega$, and, moreover, that there is a bounded set $\omega \subset \mathbb{R}^{N-M}$ such that $\Omega \subset \omega \times \mathbb{R}^{M}$ (this includes any cylindrical domain);

(iv) A union of an asymptotically contractive set and an asymptotically null set;

(v) A finite union of cylindrical domains.

The proof in all the cases is elementary. We provide only the sketches for two least trivial statements. In the case (i), by Lemma 4.4, all dislocated limits are equal zero almost everywhere on $\mathbb{R}^{N}$, and thus their trace on $H_{0}^{1}(\Omega)$ is zero. The case (iv) is proved by a partition of unity.

Corollary 4.9. The following sets are not asymptotically contractive:

(i) An open set $\Omega \subset \mathbb{R}^{N}$ (other than $\mathbb{R}^{N}$ itself) which for every $R>0$ contains a ball of radius $R$;

(ii) An open cylinder from which one has subtracted a compact subset of positive capacity; 
(iii) A product $\omega \times(0, \infty)$, where $\omega$ is an open set (in particular, a half-space).

The proof is elementary and is based on finidng a sequence $\alpha_{k}$ such that $\lim \inf \left(\Omega+\alpha_{k}\right)$ contains a closed set $Z$ with $\operatorname{cap}(Z \backslash \Omega)>0$ taking a function with support in $Z$ and tranlsating shifting it back while multiplying it with an appropriate cut-off function.

\subsection{Examples of compactness in Sobolev imbeddings}

Exmaple 1. Let $\Omega \subset \mathbb{R}^{N}, N>2$, be an open set. Due to Theorem 2.3, the imbedding of $H_{0}^{1}(\Omega)$ into $L^{p}(\Omega), p \in\left(2,2^{*}\right)$, is compact if and only if $\Omega$ is asymptotically null. Indeed, if $\Omega$ is asymptotically null, for every bounded sequence $u_{k} \in H_{0}^{1}(\Omega)$, the dislocated limits $w^{(n)}$ will be zero for all $n>1$. In other words, for an appropriate subsequence, $u_{k} \stackrel{c w}{\longrightarrow} w^{(1)}:=$ $\mathrm{w}$-lim $u_{k}$, which by Lemma 3.1 implies convergence in $L^{p}$. Conversely, if $\Omega$ is not asymptotically null, there is a sequence $\alpha_{k},\left|\alpha_{k}\right| \rightarrow \infty$, and a sequence $u_{k}$, such that

$$
u_{k}\left(\cdot+\alpha_{k}\right) \rightarrow w \neq 0 \text { in } H^{1}\left(\mathbb{R}^{N}\right) .
$$

Without loss of generality, we can assume that $u_{k} \rightarrow 0$, since we can always subtract from $u_{k}$ its weak limit while preserving (45). Then by Lemma 3.2 , the $L^{p}$-norm of $u_{k}$ for $k$ large will be bounded away from zero, implying lack of compactness.

By Corollary 4.8 the condition $\forall \alpha_{k} \in \mathbb{R}^{N},\left|\alpha_{k}\right| \rightarrow \infty, \mid \liminf (\Omega+$ $\left.\alpha_{k}\right) \mid=0$ is a sufficient condition for compactness. Related conditions have been known in literature for decades (e.g. [2],[4]; see [14] for further references and a necessary and sufficient condition of compactness in terms of capacity).

Example 2. The following result was proved (for less general domains) in $[6]$.

Let $G_{V}$ be a group of rotations in a subspace $V$ of $\mathbb{R}^{N}$ of dimension at least 2 (extended to $\mathbb{R}^{N}$ by the identity on $V^{\perp}$ ). Let $\Omega \subset \mathbb{R}^{N}$ satisfy $g \Omega=\Omega$ for all $g \in G_{V}$ and be such that for every sequence $\alpha$ with $\alpha_{k} \in \mathbb{R}^{N}$ satisfying $\sup _{k}\left|\pi_{V} \alpha_{k}\right|<\infty$ and $\alpha_{k} \rightarrow \infty$, the asymptotic set $\liminf \left(\Omega+\alpha_{k}\right)$ has measure zero. Let $H=\left\{u \in H_{0}^{1}(\Omega): u \circ g=u\right\}$. Then $H$ is compactly imbedded into $L^{p}, 2<p<2^{*}$. 
This statement can be proved as follows. We will consider the functions $u_{k}$ extended by zero to elements of $H^{1}\left(\mathbb{R}^{N}\right)$. Without loss of generalty assume that $u_{k}$ weakly converges to zero. Let $\alpha_{k} \in \mathbb{R}^{N},\left|\alpha_{k}\right| \rightarrow \infty$ and assume that

$$
0 \neq w=\mathrm{w}-\lim u_{k}\left(\cdot+\alpha_{k}\right) .
$$

If $\sup _{k}\left|\pi_{V} \alpha_{k}\right|<\infty$, then, by assumption, $\left|\liminf \left(\Omega+\alpha_{k}\right)\right|=0$ and by Lemma $4.4, w=0$ a.e., so from (46) follows that, on a renamed subsequence, $\left|\pi_{V} \alpha_{k}\right| \rightarrow \infty$. This implies in turn that if $g \in G_{V}, g \neq i d$, then $\left|g \alpha_{k}-\alpha_{k}\right|=\left|\pi_{V}(g-i d) \alpha_{k}\right| \rightarrow \infty$. Using invariance of the functions $w$ and $u_{k}$, one obtains $w=w\left(g^{-1} \cdot\right)=\mathrm{w}-\lim u_{k}\left(g^{-1} \cdot+\alpha_{k}\right)=\mathrm{w}-\lim u_{k}(\cdot+$ $\left.g \alpha_{k}\right)$.

Now, taking infinitely many distinct $g$ 's in Theorem 2.3 we get

$$
+\infty=\sum_{g}\left\|w \circ g^{*}\right\|^{2} \leq \liminf \left\|u_{k}\right\|^{2}
$$

a contradiction.

\section{Aplications to elliptic problems}

\subsection{Existence of the ground state solution on asymptoti- cally contractive domains}

We consider the following variational problem.

$$
C_{\Omega}:=\sup _{\|u\|_{H_{0}^{1}(\Omega)} \leq 1} \int_{\Omega}|u|^{p+1} d x
$$

with an unbounded open set $\Omega \subset \mathbb{R}^{N}, N>2$.

Theorem 5.1. If the set $\Omega$ is asymptotically contractive and $2<p+1<$ $2^{*}$, then the supremum in (48) is attained and the maximizer is, modulo a constant multiple, a solution to

$$
-\Delta u+u=u^{p}, u>0 \text { in } \Omega .
$$

Proof. Let $u_{k}$ be a maximizing sequence for (48). We consider the decomposition of Theorem 2.3 for $u_{k}$ as a sequence in $H^{1}\left(\mathbb{R}^{N}\right)$. Since $\Omega$ is asymptotically contractive, $\left.w^{(n)} \in H_{0}^{1}(\Omega)\right)$ by Proposition 4.2. 
Every $w^{(n)}$ has to be, modulo a constant multiple, a maximizer for (48): otherwise we replace $w^{(n)}$ in the decomposition of Theorem 2.3 by a function that gives a higher value of $\int_{\Omega}\left|u_{k}\right|^{p+1} d x$ with the same Sobolev norm. It is easy to see that the Sobolev norm of the new decomposition (in the limit, modulo an extraction) does not increase, while the value of $\int_{\Omega}\left|u_{k}\right|^{p+1} d x$ on the new sequence becomes greater than and bounded away from $C_{\Omega}$, a contradiction.

\subsection{A problem with a critical exponent on unbounded domains}

Let

$$
\lambda_{0}:=\inf _{\int_{\Omega} u^{2}=1, u \in C_{0}^{\infty}(\Omega)} \int_{\Omega}|\nabla u|^{2} .
$$

Theorem 5.2. Let $\Omega \subset R^{N}, N>3$, be asymptotically contractive and assume that $\lambda_{0}>0$. If $\lambda \in\left(0, \lambda_{0}\right)$, then the problem

$$
-\Delta u-\lambda u=u^{2^{*}-1}, u \in H_{0}^{1}(\Omega),
$$

has a positive solution.

This result is an immediate extension of the well known result [3] of Brezis and Nirenberg to the case of unbounded domain. There exists an earlier generalization by [16] which, in addition to asymptotic contractiveness, also requires that domains are asymptotically cylindric.

Proof. Consider the following variational problem:

$$
c(\Omega, \lambda)=\sup _{\int_{\Omega}|\nabla u|^{2}-\lambda \int_{\Omega} u^{2}=1} \int_{\Omega}|u|^{2^{*}}, \lambda>0 .
$$

The constant $c(\Omega, \lambda)$ is finite only for $\lambda<\lambda_{0}$. To verify solvability of (51) we will show that the maximum in (52) is attained. It is known ([3], Lemma 1.1, cf. also [12]) that

$$
c(\Omega, \lambda) \geq c\left(B_{\epsilon}, \lambda\right)>c\left(\mathbb{R}^{N}, 0\right) \text { for all } \lambda>0,
$$

where $B_{\epsilon}$ is an open ball of radius $\epsilon>0$ contained in $\Omega$. We will use this relation to exclude maximizing sequences for $c(\Omega, \lambda)$ that involve dilations of a maximizer for $c\left(\mathbb{R}^{N}, 0\right)$. The argument is as follows. Let $u_{k}$ 
be a maximizing sequence for $c(\Omega, \lambda)$ which we consider as a sequence of elements in $H_{0}^{1}\left(\mathbb{R}^{N}\right)$. We apply Proposition 4.3. Let $Q_{\lambda}(u):=\int\left(|\nabla u|^{2}-\right.$ $\lambda u^{2}$ ). Then we will have, on a renamed subsequence, with $\mathbb{N}_{1}$ as in Lemma 3.6,

$$
c(\Omega, \lambda)=\sum_{n} \int\left|w^{(n)}\right|^{2^{*}}
$$

and

$$
\sum_{n \in \mathbb{N}_{1}} Q_{\lambda}\left(w^{(n)}\right)+\sum_{n \notin \mathbb{N}_{1}} Q_{0}\left(w^{(n)}\right) \leq 1 .
$$

The latter equation requires to reproduce the argument of (16)-(19) with the square $D^{1,2}$-norm replaced by $Q_{\lambda}$. If for any $n \notin \mathbb{N}_{1}, w^{(n)}$ is not a maximizer (up to a constant multiple) for $c\left(\mathbb{R}^{N}, 0\right.$ ), then $u_{k}$ is not a maximizing sequence, since subtracting this $w^{(n)}$ from $u_{k}$ and adding instead a sequence of suitable dilations of the Talenti function, multiplied by a smooth cut-off function supported on $\Omega$, will preserve the bound (55) while increasing the value of $\int\left|u_{k}\right|^{2^{*}}$ above $c(\Omega, \lambda)$. A simple subadditivity argument shows that one would also increase the value of $\int\left|u_{k}\right|^{2^{*}}$ if one had more than one non-zero $w^{(n)}, n \notin \mathbb{N}_{1}$, which we will denote as $w^{\left(n_{0}\right)}$. If for all $n \in \mathbb{N}_{1}, w^{(n)}=0$, then we would have $c(\Omega, \lambda)=c\left(\mathbb{R}^{N}, 0\right)$, a contradiction to (53). A similar subaddiditivity argument would provide that $w^{(n)}$ will be non-zero for at most one $n \in \mathbb{N}_{1}$, say $n_{1}$, and that $\int|u|^{2^{*}}$ will not attain its maximal value unless $w^{\left(n_{0}\right)}=0$ and $Q_{\lambda}\left(w^{\left(n_{1}\right)}\right)=1$. Thus $u_{k}\left(\cdot-\alpha_{k}^{\left(n_{1}\right.}\right) \rightarrow w^{\left(n_{1}\right)}$ in $H^{1}$. Consequently, $w^{\left(n_{1}\right)}$ is the maximizer for $c(\Omega, \lambda)$.

\subsection{Symmetric ground state solution on an exterior do- main}

The following result is proved in [17], using Theorem 2.3 to verify the (PS)-condition; dislocated terms in the expansion for the Palais-Smale sequence are excluded by energy estimates.

Theorem 5.3. Let $\Omega \subset \mathbb{R}^{N}, N>2$, be a complement of a star-shaped closed set and let $f \in C(\mathbb{R})$ satisfy

$$
\begin{aligned}
\forall \epsilon>0 \exists C_{\epsilon}>0:|f(s)| \leq \epsilon|s|+C_{\epsilon}|s|^{2^{*}-1}, \\
\lim _{s \rightarrow \infty} f(s) / s=+\infty .
\end{aligned}
$$


Let

$$
T_{n}=\left(\begin{array}{ccccc}
\cos \left(\frac{2 \pi}{n}\right) & \sin \left(\frac{2 \pi}{n}\right) & 0 & \cdots & 0 \\
-\sin \left(\frac{2 \pi}{n}\right) & \cos \left(\frac{2 \pi}{n}\right) & 0 & \cdots & 0 \\
0 & 0 & 1 & \cdots & 0 \\
\cdots & \cdots & \cdots & \cdots & 0 \\
0 & 0 & 0 & \cdots & 1
\end{array}\right)
$$

Let $H_{n}=\left\{u \in H_{0}^{1}(\Omega): u \circ T_{n}=u\right\}$. Then there exists a $n_{0} \in \mathbb{N}$ dependent on $N$ and $f$ such that for every $\Omega$ satisfying $T_{n} \Omega=\Omega$ with a $n>n_{0}$, the problem

$$
-\Delta u+u=f(u) ; u \in H_{n} \backslash\{0\}
$$

has a nontrivial solution.

\section{References}

[1] D.R. Adams, L.I.Hedberg, Function Spaces and Potential Theory, Springer 1995.

[2] R.A. Adams, Compact imbedding theorems for quasibounded domains, Trans.Amer.Math.Soc., 148, 445-459 (1970).

[3] H. Brezis, L. Nirenberg, Positive solutions of an elliptic equation with a nonlinearity involving critical Sobolev exponent, Comm.Pure Appl.Math 36 437-476 (1983).

[4] C. Clark, An embedding theorem for function spaces, Pacific J.Math. 19, 243-251 (1966).

[5] J. Chabrowsky, Concentration-compactness principle at infinity and semilinear elliptic equations involving critical and subcritical Sobolev exponents, Calc.Var. 3,493-512 (1995).

[6] M. Esteban, P.-L. Lions, A compactness lemma, Nonlinear Anal. 7, 381385 (1983).

[7] M. Esteban, P.-L. Lions, Existence and non-existence results for semilinear elliptic problems in unbounded domains, Proc.Roy.Soc.Edinburgh Sect. A 93, 1-14 (1982/83).

[8] E. Lieb, On the lowest eigenvalue of the Laplacian for the intersection of two domains. Invent. Math. 74, 441-448 (1983).

[9] P.-L. Lions, The concentration-compactness principle in the calculus of variations. The locally compact case, part 1. Ann.Inst.H.Poincare, Analyse non linéaire 1, 109-1453 (1984). 
[10] P.-L. Lions, The concentration-compactness principle in the calculus of variations. The locally compact case, part 2. Ann.Inst.H.Poincare, Analyse non linéaire 1, 223-283 (1984).

[11] P.-L. Lions, The concentration-compactness principle in the calculus of variations. The Limit Case, Revista Matematica Iberoamericana, Part 1, 1.1 145-201 (1985).

[12] P.-L. Lions, The concentration-compactness principle in the calculus of variations. The Limit Case, Revista Matematica Iberoamericana, Part 2, 1.2 45-121 (1985)

[13] P.-L. Lions, Solutions of Hartree-Fock equations for Coulomb systems, Comm.Math.Phys. 109, 33-97 (1987).

[14] V.G. Maz’ja, Sobolev Spaces, Springer-Verlag (1985).

[15] M. Del Pino, P. Felmer, Least energy solutions for elliptic equations in unbounded domains, Proc. Royal Soc. Edinburgh 126A, 195-208 (1996).

[16] M. Ramos, Z.-Q. Wang, M. Willem, Positive solutions for elliptic equations with critical growth in unbounded domains, in: A.Ioffe, S.Reich, I. Shaffrir (eds), Calculus of Variations and Differential Equations, Chapman \& Hall/CRC Research Notes in Mathematics 410, 192-199 (2000).

[17] I. Schindler, K. Tintarev, Semilinear elliptic problems on unbounded domains, in: A.Ioffe, S.Reich, I.Shaffrir (eds), Calculus of Variations and Differential Equations, Chapman \& Hall/CRC Research Notes in Mathematics 410, 210-217 (2000).

[18] I. Schindler, K. Tintarev, Abstract concentration compactness and elliptic equations on unbounded domains, to appear in: M. R. Grossinho, M. Ramos, C. Rebelo, L. Sánchez (eds) Nonlinear Analysis and its Applications to Differential Equations, Birkhäuser, Boston 2001, 369-378. 
I. Schindler Ceremath,University of Toulouse I

and

UMR MIP CNRS 5640

Université Paul Sabatier

E-mail: ischindl@univ-tlse1.fr

K.Tintarev

Uppsala University

E-mail: kyril@math.uu.se

Recibido: 20 de Marzo de 2001

Revisado: 4 de Febrero de 2002 